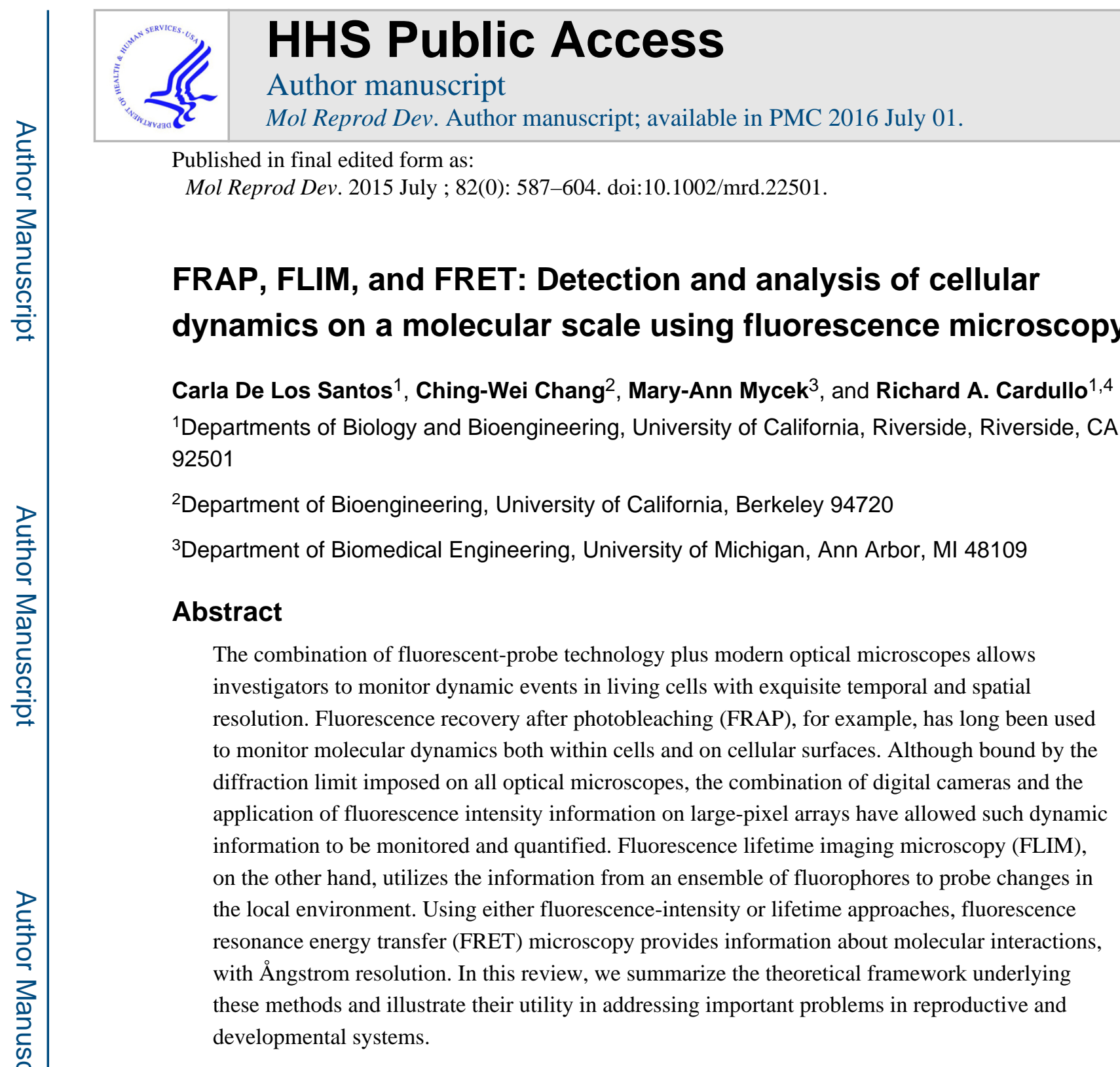

\title{
INTRODUCTION
}

Optical microscopes were originally developed to image objects that cannot be resolved with the naked eye. The introduction of histological dyes with specific physicochemical characteristics along with sophisticated lenses, polarizers, and prisms expanded the utility of microscopes by enhancing contrast and optimizing resolution close to the theoretical limits. By further optimizing the contrast and utilizing improved image-capture technologies, certain molecular events could be detected, such as the movement of vesicles along cytoskeletal elements in cells, although the structures themselves could not be resolved since they were outside the theoretical diffraction resolution limit of $\sim 200 \mathrm{~nm}$ (Goodwin, this volume).

\footnotetext{
${ }^{4}$ Corresponding Author: Richard A. Cardullo, Department of Biology, University of California, Riverside, Riverside, CA 92506, cardullo@ucr.edu, Ph: 951-827-6457.
} 
The development of fluorescent probes added another dimension to optical microscopy. The first fluorescent probes were originally developed as another class of contrast-enhancing agents that could be used by cell biologists. Many of these fluorochromes had the added advantage that they could either be chemically modified to react with a range of macromolecules, including proteins and polynucleic acids, or could modulate their fluorescence properties in response to environmental changes (e.g., $\mathrm{pH}, \mathrm{Ca}^{2+}$, membrane potential, etcetera). These fluorescent probes not only enabled localization within particular cellular compartments, but because fluorochromes operate under defined and often narrow excitation and emission wavelengths, multiple probes could be used at once with minimal spectral overlap, thus providing dynamic, low-resolution maps of living cells. With the addition of new microscopic (e.g., confocal) and computational (e.g. deconvolution) strategies to remove out-of-focus signal from fluorescent sources, the utility of these probes has become even more powerful. Yet, none of these advances allowed quantitative information to be gleaned below the optical diffraction limit of the traditional fluorescence microscope.

Fluorochromes are essentially spectroscopic probes with defining characteristics that allow them to respond to environmental conditions at molecular scales. Because of this attribute, additional information about particular fluorescent probes can be ascertained by monitoring, for instance, average ensemble changes in fluorescence intensities at specific wavelengths or the lifetimes of those fluorophores following an excitation pulse. Utilizing these properties, optical microscopes can therefore provide information well beyond the resolution limit. Indeed, fluorescence imaging systems allow both temporal and spatial information that was not previously attainable by optical microscopy, resulting in dynamic maps of processes in cells with sub-micron (and even nanometer) and nanosecond precision. Such tools are particularly useful for the developmental and reproductive biologist, who often must monitor rapid changes in cells in response to genetically programmed conditions and/or evolving environmental conditions.

In this review we will first briefly describe the spectral characteristics of fluorescent probes that can be exploited by microscopists to provide information at molecular scales. We will then describe three different microscopic methodologies that take advantage of these properties: fluorescence recovery after photobleaching (FRAP), fluorescence lifetime imaging microscopy (FLIM), and non-radiative fluorescence resonance energy transfer (FRET). FRAP provides valuable information about the mobility of molecules on surfaces and within cells, and can be used to monitor molecular assemblies and the dynamics of complex domains over time. FLIM monitors the environment around fluorophores by altering the characteristic lifetime of those molecules. And FRET directly monitors the direct interaction of two fluorophores when they come within nanometer distances of one another.

\section{ESSENTIALS OF FLUORESCENCE SPECTROSCOPY}

Before delving into the three methodologies that will be discussed in this review, it is important to understand the fundamental spectroscopic properties of fluorescent probes that can be exploited to extract information on a molecular scale. Beyond the properties that 
allow for the visible detection of specific molecules within cellular compartments, the characteristic physicochemical properties of fluorochromes allow one to monitor changes in molecular dynamics and interactions as well as changes in the local environment. It is precisely these properties that are exploited in the design and revision of many of probes that are useful for following cellular and molecular in a wide range of developmental systems.

Although many molecules absorb light at specific wavelengths, only a few are capable of fluorescence emission. Molecules that absorb light and lose energy within the singlet ground state $\left(\mathrm{S}_{0}\right)$, through vibration or rotation, are not capable of fluorescence. In contrast, fluorescent probes first absorb ultraviolet or visible light, resulting in an electron being promoted to a higher energy level (typically either $S_{1}$ or $S_{2}$ ). For those molecules that possess a large separation between the excited-state vibrational-energy levels and the ground-state vibrational-energy levels, energy is lost through a combination of vibrational relaxation to the lowest vibrational-energy level in the first excited state plus the emission of a photon as the electron returns to the ground state (Fig 1). Molecular fluorochromes therefore absorb photons of higher energy (lower wavelength) than the photons they emit, a property known as the Stokes shift. Because there is always a probability of excitation and emission at various wavelengths, a spectrum of excitation and emission wavelengths that generally mirror one another are found in a large number of fluorochromes (Lakowicz, 2006). The width of these excitation and emission spectra, along with the overlap between those spectra, necessitates the use of various filters or other wavelength-selection devices (e.g., diffraction gratings, acousto-optical devices, light emitting diodes, etc.) to more precisely control the wavelengths of light that are used for excitation or collected for emission.

Once a fluorochrome absorbs a photon and sends an electron into an excited state, the molecule can experience a number of different fates. Most frequently, the electron first returns to the lowest vibrational energy state and then emits a higher wavelength photon. This entire process typically occurs on the order of nanoseconds, with the return of the electron from the lowest vibrational state of the first excited state to the ground state constituting the rate-limiting step (in contrast, the processes of photon absorption and vibrational relaxation are on the order of femtoseconds and picoseconds, respectively). The fluorescence lifetime is, therefore, the specific fluorochrome-dependent characteristic period that the molecule spends in the excited state before decaying back to the ground state. Fluorescence lifetime reflects both the average time required for a longer-wavelength photon to be emitted following absorption of the excitation photon as well as slower competing processes that contribute to that lifetime, including solvent effects, changes in environmental parameters (e.g., $\mathrm{pH}$, ionic strength, etc.), and other possible spectroscopic transitions between the excited and ground states (Lakowicz, 2006; Chang et al., 2007). Consequently, fluorescence lifetime is a powerful and exquisitely sensitive parameter for monitoring a number of critical phenomena, including intra- and intermolecular associations as well as probing both intra- and extracellular environments.

There are two important ramifications of having a fluorochrome in a transient excited state. First, the excited molecule experiences a charge polarization that results in the creation of an electric dipole. This dipole can then interact with other molecules, at which point it can 
either lose energy by donating its emitted photon to those molecules or by non-radiatively inducing a dipole on another nearby molecule. In the rare case when there is a direct absorption of an emitted photon by another fluorophore, there is no change in the lifetime of the fluorochromes. In the case of a dipole-induced dipole interaction, however, the resulting loss in energy manifests itself as a decrease in donor fluorescence lifetime as well as a quenching of donor fluorescence intensity and the appearance of the acceptor molecule fluorescence emission, known as "sensitized emission". This non-radiative process, known as FRET (originally for Förster resonance energy transfer, but more commonly for fluorescence resonance energy transfer), is useful to microscopists and will be expanded on later in this review. Another consequence of the excited state is the molecule's possible transition into a transient, excited triplet state, which is longer-lived and allows the fluorophore to react with other molecules in the environment, including molecular oxygen (which is a triplet in its ground state); if the free radical reacts, the electronic resonance configuration of the fluorochrome is altered and the photon emission is quenched. Irreversible quenching, resulting from the covalent modification of a fluorochrome, results in a condition known as photobleaching, which is the basis for FRAP.

\section{FLUORESCENCE RECOVERY AFTER PHOTOBLEACHING}

\section{The fundamentals}

A number of techniques have been developed to monitor the behavior of molecules in solution and in cells that represent, for example, molecular interactions, molecular mobility, and chemical kinetics. Three of the methodologies that are commonly employed include single molecule tracking, (SMT), fluorescence correlation spectroscopy (FCS), and FRAP. SMT methods allow the direct measurement of biological events at the level of single fluorescent molecules, without interference from the average signal from a surrounding ensemble of fluorescent molecules (Martin-Fernandez and Clarke, 2012; Kusumi et al., 2014). Similarly, FCS is a powerful, but complex, technique that measures the spatialtemporal correlation of individual molecules with themselves; this method complements single-molecule measurements (Elson, 2011). Both SMT and FCS have proven to be powerful tools for probing cellular domains and both are critical to the development of super-resolution microscopy methods. Unfortunately, both methods require specialized equipment and expertise and are generally applicable when the concentration of very bright fluorophores is small (Martin-Fernandez and Clarke, 2012). As a result, FCS and SMT are extremely powerful techniques for monitoring diffusion at very low fluorophore concentration. In contrast, FRAP is an ensemble method that is applicable for a wide range of biological phenomena utilizing physiologically relevant concentrations of fluorophores.

FRAP, originally introduced as "fluorescence photobleaching recovery", is commonly used for studying the dynamics of fluorescent molecules in living cells (Cardullo et al., 1991; Mullineaux and Kirchoff, 2007; Carisey et al., 2011). The technique was initially used to monitor the lateral diffusion of fluorescent probes on cell surfaces (Schlessinger et al., 1976; Axelrod et al., 1976a; Koppel et al., 1976) - e.g. to quantify the lateral diffusion coefficient of acetylcholine receptors in developing chick myotubes (Axelrod et al., 1976b). Subsequent studies used the technique to monitor the mobility of both membrane lipids and proteins in a 
variety of cell types (Schlessinger et al., 1977; Lakowicz, 2006; Mullineaux and Kirchoff, 2007; Waters, 2007). The technique is now routinely used to qualitatively and quantitatively monitor molecular dynamics both on cell surfaces and within the cytoplasm. For example, FRAP has been used to measure the rate and extent of molecular mobility, the presence of binding interactions, and how these properties are affected with different treatments. These kinetics data are then quantified by analyzing the resulting fluorescence-intensity recovery curve versus time (Waters, 2007).

As described previously, fluorescent molecules are generally susceptible to photobleaching, which, even under low-excitation light intensities in a fluorescence microscope, results in the signal fading over time. Photobleaching is dramatically accelerated as the excitation light intensity increases; in the presence of an intense laser source, complete photobleaching can occur on the order of milliseconds. FRAP takes advantage of the rapid and irreversible photobleaching of fluorescently labeled molecules within a region of interest using a laser tuned to an appropriate wavelength for short durations of time (typically less than $20 \mathrm{msec}$ ). Following the light pulse, the fluorescence intensity is monitored in an area around and including the region of interest using a low-intensity light source to monitor the movement of photobleached and fluorescent molecules over time (Fig 2). FRAP yields two quantitative parameters: the percent recovery measures the fraction of molecules in the photobleached area that are mobile while the recovery-rate constant is a measure of the speed at which molecules move in and out of the bleach area (this rate constant is related to the diffusion coefficient). The recovery-rate constant can also yield information related to binding interactions within the region of interest, with different rates indicating molecular interactions within that region.

Originally, FRAP involved the use of a photomultiplier tube that quantified the recovery as average fluorescence intensity over time within the bleach area and restricted to the region of interest, necessitating the derivation of a best-fit equation to recovery data based on basic diffusion theory that allowed for accurate determinations of diffusion coefficients and percent recovery (Axelrod et al., 1976a; Koppel et al, 1976). With the advent of video (and then digital) cameras coupled with image processing methodologies to collect fluorescence intensity data over large areas, researchers were able to monitor events over extended distances and longer periods of time, allowing them to look at processes of interest to cell and developmental biologists (Cardullo et al., 1991). The availability of green fluorescence protein (GFP) and its analogs has now made FRAP widely available to a range of biologists who want to study protein dynamics in living cells without having to extrinsically label proteins of interest (Axelrod et al., 1978; Hiraoka et al., 1987; Mullineaux and Kirchhoff, 2007).

\section{Limitations of FRAP}

The possibility of photoswitching has been proposed as a limitation to the use of FRAP to determine molecular mobility. Photoswitching is a form of reversible photobleaching, where instead of the fluorescent molecule losing its fluorescence permanently, the fluorescent molecule can regain its fluorescence after a period of time. The time that it takes to regain fluorescence may interfere with the recovery period when the rate of diffusion is measured 
during FRAP. Photoswitching has been reported for GFP and its derivatives - CFP, YFP, mCherry, TagRFP, and mTFP - although a variety of studies report a $₫ 5 \%$ chance that photoswitching will occur under routine experimental conditions (Dayel et al., 1999; Sinnecker et al., 2005; Mueller et al., 2012).

Another concern with FRAP is the possibility of localized heating by the laser. For this reason, the bleaching event itself, which occurs using a high-power light source, is generally restricted to $20 \mathrm{msec}$ or less, thereby minimizing localized heating (Axelrod, 1977). Heating effects are minimized when following membrane dynamics since the aqueous reservoir surrounding the photobleached fluorophores (restricted to the narrow depth of focus of the surface) is sufficient to absorb the effects the heating over short periods of time, but can pose a potential problem in the cytoplasm, where the laser profile impacts a larger volume.

Although useful for many applications related to developmental biology, care must be taken to fully understand all of the parameters that affect reproducible recovery behaviors in or on cells. Information about the bleach geometry, bleach depth, bleach pulse time, and bleach volume can significantly alter results, especially when determining quantitative values of diffusion coefficients and percent recoveries (Davoust et al., 1982; Munnelly et al., 1998). When working over long time periods under changing cellular conditions, such as during development, it is imperative that the experimental and imaging parameters remain unchanged so that accurate comparisons can be ascertained. Such considerations are particularly important when confocal microscopes are used in photobleaching and photoactivation studies within cells due to the complex hourglass geometry of the bleach profile in the $z$-direction, which is difficult to model, resulting in confocal determinations of molecular dynamics in cells a qualitative, but not a quantitative, exercise. An exception is the use of two-photon FRAP, which provides a well-defined bleach volume that yields accurate diffusion measurements in three dimensions (Brown et al., 1999; Waharte et al., 2005).

\section{FRAP microscope setup}

The components of a FRAP experimental setup include: a fluorescence microscope (epifluorescence or confocal), a laser, an additional light source (when cameras are used instead of photomultiplier tubes), a fluorescence intensity detector, and the use of an appropriate fluorescent probe (Mullineaux and Kirchhoff, 2007; Waters, 2007; Carisey et al., 2011). In many cases, and when it is practical, a confocal microscope is preferred because out-of-focus light is removed from the resulting image (Waters, 2007), although the use of a confocal in three-dimensional situations makes precise quantitation of diffusion coefficients unrealistic due to the hourglass shape of the bleach profile (see previous section).

In early FRAP microscopes, photomultiplier tubes (PMTs) were used to measure the fluorescence intensity as a function of time within the bleached spot (Lakowicz, 2006). A PMT setup requires the light source to be a laser that can switch between high (photobleaching) and low (monitoring) intensities (Mullineaux and Kirchhoff, 2007). With the development and availability of charged-coupled devices (CCDs), however, current FRAP microscopes include a CCD in place of the PMT and the imaged region of interest 
includes areas beyond the original bleach spot (Hiraoka et al., 1987). An advantage of employing CCDs over PMTs is that signal intensity data can be collected on an entire image, allowing dynamic variations in fluorophores mobility inside and outside of the bleached area to be routinely monitored, yielding a more complete picture of molecular structures and processes (Cardullo et al., 1991).

A wide range of fluorescent probes have been and are still utilized in FRAP studies. The advent of genetically encoded GFP and fluorescent-protein analogs, however, now make these probes of choice for cellular studies (Sprague et al., 2004; Carisey et al., 2011).

\section{Applications of FRAP in reproductive and developmental biology}

FRAP has been applied to many developmental studies, including the original studies monitoring the behavior of acetylcholine receptors in chick myotubes (Axelrod et al., 1976b); the extent of lipid mobility in gametes (Wolf and Voglmayr, 1984; Wolf et al., 1986; Wolf et al., 1988); the distribution and dynamics of galactosyltransferase on the surface of mouse sperm (Cardullo and Wolf, 1995) and ADAM1/ADAM2 on guinea pig sperm (Hunnicutt et al., 2008); and the formation of an acrosomal matrix during spermatogenesis in water strider sperm (Fig 3) (Miyata et. al., 2011). Each of these applications are detailed below.

The dynamics of acetylcholine receptors was studied in chick to qualitatively and quantitatively characterize receptor appearance, mobility, and clustering during development (Axelrod et al., 1976; Axelrod et al., 1978; Dubinsky et al., 1989). The qualitative results were used to identify populations of receptors that were either mobile or immobile (Axelrod et al., 1978; Dubinsky et al., 1989). Quantitatively, FRAP was used to identify differences in diffusion coefficients and percent recoveries within the mobile acetylcholine receptor populations.

FRAP has also been used to monitor the distribution and dynamics of surface $\beta-1,4-$ galactosyltransferase (GalTase) on mouse sperm under different conditions. Sperm surface GalTase has been identified as a possible zona pellucida receptor that is required for successful fertilization. FRAP was used to monitor the redistribution of GalTase on the sperm surface from the posterior head to the anterior head of mouse sperm, using different monovalent probes that were specific for GalTase under conditions when the enzyme was either cross-linked with bivalent antibodies or not cross-linked in the presence of antiGalTase Fab fragments (Cardullo and Wolf, 1995). This study concluded that cross-linking mouse sperm surface GalTase was sufficient to induce its redistribution with a corresponding decrease in the percent recovery, without a change in the diffusion coefficient of the mobile fraction (Cardullo and Wolf, 1995). In a similar study, the presence of dbcAMP was found to increase the mobility of the heterodimeric guinea pig sperm membrane protein ADAM1/ADAM2 (fertilin) more than tenfold in cauda epididymal sperm but not testicular sperm. This result was similar to those found when cauda epididymal sperm were exposed to capacitating conditions (Hunnicutt et al., 2008).

FRAP has also been used to monitor the incorporation of a flavin adenine dinucleotide (FAD)-associated protein into the acrosomal matrix of sperm from the water strider, 
Aquarius remigis, during spermatogenesis and spermiogenesis (Fig 3) (Miyata et al., 2011). Along with fluorescence polarization microscopy, this study demonstrated that, following meiosis, the FAD-containing protein rapidly accumulates into a rigid matrix that ultimately extends as a $\sim 2500$ micron helical structure from base to tip. Prior to its incorporation into the matrix, the fluorophores are freely mobile, but they become increasingly less mobile as spermiogenesis proceeds (Miyata et al., 2011; Ott et al., 2015).

\section{FLUORESCENCE LIFETIME IMAGING MICROSCOPY}

\section{The principles}

Fluorescence lifetime is an important molecular characteristic measured in many

fluorescence spectroscopy and imaging studies, and has recently been applied to investigate biological processes at the molecular, cellular, and tissue levels (Lloyd et al., 2013a; Lloyd et al., 2013b). Fluorescence lifetime refers to the average time electrons spend in the excited state following the fluorophore's absorption of an excitation photon. Fluorophores in the first excited state $\left(S_{1}\right)$ can relax to the ground state in several different ways, as illustrated by the Jablonski diagram (Fig 1). The overall rate at which the number of excited fluorophores, $N(t)$, decreases is proportional to the sum of the non-radiative decay rate, $k$, and the radiative decay rate, $\Gamma$, as well as $N(t)$, itself:

$$
\frac{d N(t)}{d t}=-(\Gamma+k) N(t)
$$

This first-order decay behavior results in an exponential time dependence of the population in the excited state:

$$
N(t)=N_{o} e^{-(\Gamma+k) t}=N_{o} e^{-t / \tau}
$$

where the fluorescence lifetime, $\tau$, is defined as:

$$
\tau=\frac{1}{\Gamma+k}
$$

Any process that affects $k$ or $\Gamma$ will have an impact on the value of the fluorescence lifetime. A change in the fluorescence lifetime value measured experimentally can report the influence of these processes (Fig 1) -which include dynamic quenching and FRETproviding an opportunity to probe a fluorophore's microenvironment. On the other hand, fluorescence lifetime is an intrinsic property of the molecule itself, so it is generally insensitive to intensity-based artifacts in fluorescence experiments, such as optical loss, focus drift, and instability of the excitation source (Chang et al., 2007).

When more than one type of fluorophore is present (e.g., different fluorophores with distinct intrinsic lifetimes) or the same fluorophore is present in different molecular states (e.g., binding states), the resulting fluorescence will exhibit combined characteristics, giving rise to multi-exponential fluorescence decay. When analyzing mixed fluorescence lifetime data, 
the individual fluorescence decays can be separated and their relative contributions quantified (Lakowicz, 2006).

\section{Fluorescence lifetime imaging}

FLIM produces spatially resolved images of fluorescence lifetime, providing another dimension of information (temporal) for visualizing fluorophores as well as an additional source of contrast for fluorescence-imaging applications (Chen et al., 2013). FLIM can be implemented in two different experimental domains: time and frequency. The more-intuitive time-domain implementation directly resolves the fluorescence decay waveform following a temporal pulse of excitation light. To measure the frequency domain, on the other hand, continuous sinusoidally modulated excitation and emission light are analyzed to resolve fluorescence lifetime. Both time- and frequency-domain methods (which are equivalent and related by the Fourier transform) have comparable temporal resolution and discrimination, and are compatible with widefield or raster-scanned microscope designs. Choosing which implantation to use, however, depends on the particular application: frequency-domain FLIM is usually better for multi-exponential decay analysis whereas time-domain FLIM is considered more flexible for large temporal ranges, and is especially apt for long-lifetime measurements (Chang et al., 2007). Comparisons between time-domain FLIM and frequency-domain FLIM are further detailed below and summarized in Table 1.

Time-gated FLIM (Fig 4A) is one way to achieve time-domain FLIM with both rapid data acquisition and fast image processing. The instrumentation needed for time-gated FLIM (Fig 4A, left panel) separates the light beam from a pulsed laser into two parts by a beam splitter: one part travels to the microscope and serves as the sample excitation light, while the other part serves as a reference. Once the reference light beam is received by the photodiode, it is converted into an electronic signal to mark time. After a delay relative to the receipt of the reference signal, gating of the imaging detector is triggered. By varying the timing delay, a series of the fluorescence emission images following the excitation pulse can be recorded, and the fluorescence decay curves can be reconstructed for each pixel in the image (Fig 4A, right panel). The lifetime value of the fluorochrome can then be determined pixel-by-pixel using different methods. With single-exponential decay, rapid lifetime calculations can be performed with a minimum of two detection gates (Bugiel et al., 1989; Wang et al., 1991; Sharman et al., 1999), although more gates can be used to enhance data robustness relative to the noise (Cubeddu et al., 2002; Chang et al., 2007; Zhong et al., 2007; Chang and Mycek, 2010a; Chang and Mycek, 2010b; Chang and Mycek, 2012a; Chang and Mycek, 2012b). For multi-exponential decays, the data can be fitted non-linearly with least-squares or maximum likelihood methods (Chang et al., 2009).

Another useful and commonly implemented experimental approach to time-domain FLIM is via time-correlated single photon counting (TCSPC) (Bugiel et al., 1989; Ghiggino et al., 1992; Böhmer et al., 2001; Becker et al., 2002). In this approach, the fluorescent sample is excited by a relatively weaker light source, such that the rate of detecting emitted single photons is only $1-2 \%$. The timing at which these single photons are received is then recorded to build up a histogram, which represents the fluorescence decay waveform (see equations (1) and (2)). This is performed at every single pixel to reconstruct a FLIM image. 
The reconstruction of TCSPC images often involves non-linear least-squares fitting since tens to hundreds of points on the decay curve (i.e., bins in the histogram) are acquired. This implies higher robustness in multi-exponential lifetime determination processes compared to time-gated FLIM, since decay curves are better resolved temporally, but comes with the caveat of longer data acquisition time, especially since it is usually implemented with rasterscanned microscope. Interestingly, recent studies pointed out the feasibility of widefield TCSPC with potentially much higher data acquisition speeds (Hirvonen, et al., 2014). The TCSPC-decay curve-fitting process still requires significant analysis time, but "virtual" gating could be applied to greatly reduce the time required while also improving the precision of lifetime determination (see below; readers interested in more details on TCSPC data analysis, including the correction of potential decay curve distortion, are referred to book chapters by Becker (2005); Lakowicz (2006); and Chang et al. (2007)).

Frequency-domain FLIM designs are compatible with widefield microscopy (Fig 4B) as well as confocal microscopy (Booth and Wilson, 2004). The excitation light source is sinusoidally-modulated, resulting in sample emission that is sinusoidally-modulated at the same frequency, but shifted in phase with a reduced modulation depth (i.e., demodulation, or $M(x)$ in Figure 4B, with a value of $0-1$ ). Both phase shift and demodulation can be used to calculate fluorescence lifetime, with different lifetimes exhibiting different optimal modulation frequencies. Multiple (>10) modulation frequencies are usually required to better resolve multi-exponential fluorescence decay, unless the "phasor" approach is used. As a general rule, the optimal frequency is approximately the reciprocal of the lifetime. For example, typical biological fluorophores, such as cellular $\mathrm{NAD}(\mathrm{P}) \mathrm{H}$ and extracellular collagen, with lifetimes of few nanoseconds, require excitation sources modulated at 1-200 $\mathrm{MHz}$ (Chang et al., 2007). For samples exhibiting multi-exponential decay in frequencydomain FLIM, on the other hand, modulation lifetimes at a single frequency are longer than phase lifetimes. This can be understood by analyzing the data with the "polar plot" (or "phasor") method, which has been used as a visual aid for frequency-domain FLIM, and has been reported for single-frequency measurements of samples exhibiting multiple fluorescence lifetime (Clayton et al., 2004; Redford and Clegg, 2005). The analysis is performed by observing clustering of pixels in specific regions of the phasor plot, providing a global view of FLIM images. Since every molecular species is located in distinct regions on the phasor plot, they can be easily identified. Thus, this analysis approach makes the interpretation of FLIM data accessible to non-experts (see Figure 8 for an example).

\section{Challenges of FLIM}

Recently, advanced approaches to FLIM analysis have been developed to address specific challenges inherent to biological imaging applications. In live-cell FLIM applications, video-rate or low-light imaging may be required to capture the dynamics of interest or to reduce potential perturbations to living cells; unfortunately, the precision of FLIM measurements may be compromised under such conditions. One way to circumvent this problem in time-gated FLIM is to optimize the gating parameters, including both the timing and the temporal-width of the gating (Sharman et al., 1999; Elson et al., 2004; Chang and Mycek, 2010a; Chang and Mycek, 2010b). Interestingly, optimal gating can also be applied to TCSPC FLIM, utilizing virtual gating to improve the precision of recovered fluorescence 
lifetimes as well as to significantly shorten the data processing time (Chang and Mycek, 2010b). As for frequency-domain FLIM, optimization has also been developed for highthroughput applications (Esposito et al., 2007).

While optimal (virtual) gating improves FLIM in the temporal dimension, image noise removal (or "denoising") takes advantage of the spatial correlation of image pixel intensities. Wavelet analysis is a commonly employed method used for restoring fluorescence microscopy images (Vonesch, 2009; Vonesch and Unser, 2009) and for denoising frequency-domain confocal and widefield FLIM images (Buranachai et al., 2008; Spring and Clegg, 2009). For time-domain FLIM, novel total variation-based denoising methods have been applied to both time-gated and multi-photon TCSPC FLIM. These approaches have been compared with wavelet-based denoising methods, and have shown superior results (Chang and Mycek, 2012b); indeed, a multi-fold FLIM precision improvement is achieved without compromising the accuracy of measurements, and even greater improvement of FLIM can be achieved when used in combination with optimal (virtual) gating (Chang and Mycek, 2010a; Chang and Mycek, 2010b; Chang and Mycek, 2012a; Chang and Mycek, 2012b).

\section{Biological applications}

FLIM has been used to address a number of cell biology questions involving the intracellular environment. For example, this approach was used to report $\mathrm{pH}$ dynamics in living cells (Sanders et al., 1995; Lin et al., 2003), suggesting that FLIM can measure the intracellular $\mathrm{pH}$ of resting cells, and follow the $\mathrm{pH}$ fluctuations inside the cells after environmental perturbations. Measurement of dissolved oxygen concentration by FLIM in single living cells has also been demonstrated (Gerritsen et al., 1997; Sud et al., 2006b), based on the fact that the fluorescent probe can be dynamically quenched by local oxygen, thereby shortening its fluorescence lifetime (Fig 1). Such an approach has been extended to image oxygen levels in poly(dimethyl siloxane) bioreactors containing living C2C12 mouse myoblasts, where FLIM provided a quantitative measurement with high spatial resolution for monitoring oxygen in continuous cell culture systems (Sud et al., 2006a). Protocols using cell lysate-FLIM measurements have also been developed to correct the in vitro calibration of a fluorescent oxygen sensor and to improve the accuracy of the method for intracellular oxygen sensing (Sud and Mycek, 2009). FLIM has also been employed to image NADH distribution in live progenitor stem cells (Wright et al., 2012). When FLIM images are acquired at multiple wavelengths - i.e. multispectral FLIM (Becker et al., 2007; Owen et al., 2007; Lloyd et al., 2010) - functional information from multiple fluorophores can be analyzed simultaneously. With this technique, NAD(P)H and FAD, two biologicallyrelevant molecules related to cellular metabolism, can be preferentially detected with emission less than $\sim 500 \mathrm{~nm}$ for $\mathrm{NAD}(\mathrm{P}) \mathrm{H}$ and greater than $\sim 500 \mathrm{~nm}$ for FAD (Skala et al., 2007).

Multi-photon FLIM has been used for in vivo detection of hyperactivity and intercellular calcium waves in astrocytes from a mouse model of Alzheimer's disease (Fig 5) (Kuchibhotla et al., 2009). Label-free, multi-photon FLIM was also employed to characterize normal human epidermis at different tissue sites in patients of different ages 
(Benati et al., 2011) and to monitor the metastasis and the microenvironment of mammary tumors (Provenzano et al., 2009); both applications demonstrate FLIM's potential clinical utility. Indeed, a recent study employed label-free multi-photon FLIM to non-invasively characterize cellular viability in engineered tissues manufactured with primary human cells (Chen et al., 2014), demonstrating the potential of FLIM to address an unmet regulatory need in regenerative medicine. Another major biological application of FLIM involves the detection of FRET events.

\section{FLUORESCENCE RESONANCE ENERGY TRANSFER MICROSCOPY}

\section{The theory}

The theory underlying FRET was developed by Theodor Förster (Förster, 1948). The method is a commonly used for measuring the spatial distance between two (or multiple) fluorophores. FRET can be considered an in vivo "nanoscale ruler", since the distance for FRET to occur is usually within several nanometers, which is comparable to the size of many proteins, the distance within which proteins interact, the thickness of biological membranes, and the distance between sites on multi-subunit proteins. Therefore, FRET can report the distance between two distinct sites on a macromolecule, the distance between two fluorophore-tagged proteins, and hence if and how these two proteins interact in a live cell (Cardullo, 2007; Chang, 2009).

FRET occurs when the emission spectrum of a FRET donor overlaps with the excitation spectrum of the FRET acceptor. After donor excitation, non-radiative energy transfer occurs if the donor-acceptor pair is in close proximity (i.e., the "non-radiative decay pathway" illustrated in Figure 1), as a result of long-range dipole-dipole interaction between the pair of molecules (Fig 6). While almost all current applications of FRET involve only one FRET pair, there have been developments in the analysis of multiple FRET pairs, which has the potential to simultaneously study interactions between components inside a protein complex (Sun et al., 2010; Hoppe et al., 2013).

Genetically encoded fluorophores, most likely fluorescent proteins, are commonly used for live-cell FRET applications. Fluorescent-protein FRET probes can be made to detect the conformational change of a full-length protein (Hao and Macara, 2008); protease cleavage (Buranachai et al., 2008; Ouyang et al., 2008); binding of protein sensory domain with its ligand (Kalab et al., 2002; Nguyen and Daugherty, 2005; Shimozono et al., 2006; Kolossov et al., 2008); kinase and small GTPase activity (Miyawaki et al., 1997; Nakamura et al., 2006; Zhang and Allen, 2007; Zhong et al., 2007; Nakaya et al., 2008; Chang et al., 2009; Machacek et al., 2009; Chang and Mycek, 2012a); and even the movement of a protein sensory domain (Sakai et al., 2001; Tsutsui et al., 2008) (Some of these applications are reviewed by Kalab and Soderholm (2010)). FRET probes with an elastic linker connecting the donor and accepter have recently made it possible to study molecular tension in the context of cell mechanics and mechano-transduction with high spatial and temporal resolution (Meng et al., 2008; Grashoff et al., 2010; Meng and Sachs, 2011; Murakoshi et al., 2011; Verma et al., 2012; Chang and Kumar, 2013; Conway et al., 2013). 
To determine how to choose the best FRET probes for a specific application, it is important to understand the factors that will affect FRET. FRET efficiency, $E$, is defined as the fraction of energy the donor receives during excitation that is transferred to the acceptor:

$$
E=\frac{R_{o}^{6}}{R_{o}^{6}+R_{A-D}{ }^{6}}
$$

where $R_{O}$ is the Förster distance, at which the FRET efficiency is $50 \%$, and $R_{A-D}$ is the acceptor-donor distance. From the above equation, it can be clearly seen that the dependence of $E$ on $R_{A-D}$ is highly non-linear and strongest when the donor-acceptor distance is near $R_{0}$; when $r=2 R_{0}$, the energy transfer efficiency drops to only $1.56 \%$.

There are a few factors that affect $R_{0}$, which in turn affect FRET efficiency when the donoracceptor distance is fixed (Förster, 1948). One obvious factor is the selection of the FRET pair, particularly the overlap of the donor emission and acceptor excitation spectra. Researchers optimize FRET pairs using different fluorophores as well as variants of the same fluorophore or with various linker sizes (Komatsu et al., 2011; Lam et al., 2012; Fritz et al., 2013). Currently, the most commonly used FRET pairs are CFP-YFP variants, mTFP1-YFP variants, and GFP-RFP variants. Recently, the relative orientation of the FRET pair has been given more attention due to its direct impact on $R_{0}$. For example, intramolecular FRET exhibits some non-zero background FRET signal since the donoracceptor pair is kept at a certain distance just outside the sensitive range of FRET; in theory, this background can be completely removed if the donor-acceptor dipole orientation is perpendicular. Optimization of FRET probes based on fluorophore orientation, e.g. by taking advantage of protein circular permutation, has been studied by several research groups (Meng and Sachs, 2012; Fritz et al., 2013), while other FRET probes that are based solely on fluorophore orientation rather than distance have also been developed (Meng and Sachs, 2012).

FRET is one of the factors that can influence fluorescence lifetime since the donor lifetime decreases while acceptor lifetime increases when intermolecular energy transfer occurs. One of the most favorable advantages of FLIM-based FRET detection is that only donor lifetime needs to be determined to quantify the FRET response (Pelet et al., 2006; Chang et al., 2007; Zhong etal., 2007; Chang et al., 2009; Padilla-Parra and Tramier, 2012). While this is similar to fluorescence intensity-based FRET detection -wherein the donor fluorescence intensity decreases when FRET occurs- overall donor emission can easily be affected by donor concentration, possible bleed-through from the acceptor, as well as other intensitybased artifacts (see "Fluorescence Lifetime: The Principles"), none of which influence donor fluorescence lifetime (Pelet et al., 2006; Chang et al., 2007; Zhong et al., 2007; Chang et al., 2009; Padilla-Parra and Tramier, 2012).

FLIM-based FRET detection is now commonly used in cell biology (Maeder et al., 2007; Murakoshi et al., 2011; Lin et al., 2012; Liu et al., 2012; Stirnweiss et al., 2013; Zimmermann et al., 2013). One example involves the detection of GRB2 dimerization in the cell and on the plasma membrane (Fig 7), with fluorescence-lifetime histograms clearly distinguishing the dimer and monomer subpopulations (Lin et al., 2012). FRET detection 
can be performed in either time-domain or frequency-domain FLIM, and the phasor approach to FRET analysis and detection based on frequency-domain FLIM has recently been developed (Hinde et al., 2012), providing visualization of high-FRET and low-FRET components (Fig 8).

\section{Applications of FRET}

Because of its exquisite sensitivity for detecting molecular interactions on the order of Ångstrom distances, FRET has been useful for monitoring a number of developmentally regulated events in single cells, tissues, and organs. Some examples that leverage the power of FRET are described below.

FRET is particularly useful for detecting associations between proteins in live cells. In neonatal rats, for example, FRET was used to elucidate the mechanism of dopamine uptake in mouse hippocampal neurons (Egaña et al., 2009). Previous work had established that dopaminergic transmission is terminated by dopamine uptake in the brain by a dopamine transporter (DAT), but the mechanism for synaptic vesicle uptake was unknown. Using standard immunolocalization methods, it was found that both DAT and the synaptic vesicle protein synaptotagmin-3 co-localized to the post-synaptic terminals isolated from mouse striatum. To see if this association could be observed in live neurons, CFP-labeled DAT (donor) and YFP-labeled synaptotagmin-3 (acceptor) were transfected into cultured hippocampal neurons. Using FRET microscopy, it was established that there is a physical interaction between the amino termini of DAT and synaptotagmin-3, and that this close association is essential for dopamine uptake (Egaña et al., 2009).

FRET can also be used for observing conformational changes in proteins or protein complexes within cells (Fig 9). In transgenic zebra fish, FRET microscopy was successfully used to monitor conformational changes in the $\mathrm{Ca}^{2+}$ binding domain (CBD1) of the $\mathrm{Na}^{+} /$ $\mathrm{Ca}^{2+}$ exchanger (Xie et al., 2008). These investigators expressed CBD1 (amino acids $371-$ 508) and labeled the amino- and carboxyl-termini with YFP and CFP, respectively (YFPCBD1-YFP). This construct undergoes conformational changes upon binding $\mathrm{Ca}^{2+}$, so using FRET microscopy, as well as other constructs with site-specific mutations that decrease $\mathrm{Ca}^{2+}$ binding affinity to the $\mathrm{Ca}^{2+}$-binding domain, they could measure the corresponding abolishment of $\mathrm{Ca}^{2+}$-induced FRET in YFP-CBD1-YFP (Ottolia et al., 2004; Xie et al., 2008). Real-time FRET imaging allowed the investigators to follow heart contractions in 2day-old embryonic zebrafish, and observe an increase in YFP-CBD1-YFP FRET signal during diastole, when cytoplasmic $\mathrm{Ca}^{2+}$ was decreased, and a decreased FRET signal during systole, when cytoplasmic $\mathrm{Ca}^{2+}$ increased; these changes were not observed for the mutant constructs with decreased $\mathrm{Ca}^{2+}$ binding affinity (Xie et al., 2008). Additional studies using FRET microscopy in this system showed that treating the embryonic zebrafish hearts with the ionotropic agents ouabain and isoproterenol increased both the $\mathrm{Ca}^{2+}$ transients and the changes in FRET signal (Fig 9) (Xie et al., 2008).

A recent example using FRET to measure conformational changes in a receptor was reported by two different groups working on the abscisic acid (ABA) receptor in plants. $\mathrm{ABA}$ is a stress hormone that, in times of water scarcity, can initiate a response to close the leaf stomata (Jones et al., 2014, Waadt et al., 2014). Both groups designed ABA receptor 
reporters that function at physiologic ABA concentrations, and employed them to measuring differences in ABA responses among distinct tissues in Arabidopsis (Waadt et al., 2014). Such exquisite sensitivity is a hallmark of FRET sensors, which allows dynamic molecular associations and changes to be monitored on short time scales.

FRET has also proven to be a powerful tool when coupled with sensors that detect changes in second messenger levels. An elegant example of such an application comes from the demonstration of how follicular cells regulate the prophase-to-metaphase transition in mouse oocytes in response to luteinizing hormone ( $\mathrm{LH})$ following meiotic arrest (Norris et al., 2009). These investigators developed both high-affinity cAMP and cGMP FRET sensors using YFP/CFP donor/acceptor pairs. After characterizing these FRET sensors in a fluorescence spectrometer, FRET measurements were made in follicle-enclosed oocytes using a confocal microscope to monitor changes in YFP and CFP fluorescence intensities, which were used to calculate changes in YFP/CFP emission ratios at the oocyte equator in response to microinjection of either cAMP or cGMP. These studies conclusively showed that, in response to LH, cGMP levels decrease in follicle-enclosed oocytes that have not yet undergone germinal vesicle breakdown, thus triggering an increase in the activity of the primary cAMP phosphodiesterase PDE3A that quickly depleted cAMP levels to concentrations that inhibit PKA activity (Norris et al., 2009). The exquisite sensitivity of these FRET sensors allowed these investigators to determine that a decrease in cGMP in mouse oocytes from $\sim 1 \mu \mathrm{M}$ to $\sim 40 \mathrm{nM}$ resulted in an approximately fivefold increase in PDE3A activity, which decreased the cytosolic cAMP concentration from $\sim 700 \mathrm{nM}$ to 140 $\mathrm{nM}$ and initiated the resumption of meiosis.

\section{SUMMARY}

Knowledge of the spectroscopic characteristics of fluorescent probes have provided a foundation for new molecular-level techniques that take advantage of the influence that local environment has on the behavior of these molecules. Combined with recent developments in imaging technology, these fluorochrome properties provide powerful tools for research in cellular and developmental biology. Specific methodologies - including FRAP, FLIM, FRET, and a number of other emerging technologies - allow investigators to make detailed observations and quantitative measurements in living cells far beyond the capabilities of standard fluorescence or confocal microscopes.

\section{Acknowledgments}

Grant information:

Grant Sponsor: NIH, grant number: R01 HD27244

Grant Sponsor: NIH, grant number: R01 DE019431

Grant Sponsor: Whitaker Foundation for Biomedical Research

This work was supported in part by grants from the U.S. National Institutes of Health (R01-DE-019431, to M.-AM and R01-HD-27244 to RAC) and the Whitaker Foundation for Biomedical Research (to RAC). 


\section{Abbreviations}
FLIM fluorescence lifetime imaging microscopy
FRAP fluorescence recovery after photobleaching
FRET fluorescence resonance energy transfer

\section{References}

Axelrod D. Cell surface heating during fluorescence photobleaching recovery experiments. Biophys J. 1977; 18:129-131. [PubMed: 851570]

Axelrod D, Koppel DE, Schlessinger J, Elson EL, Webb WW. Mobility measurement by analysis of fluorescence photobleaching recovery kinetics. Biophys J. 1976a; 16:1315-1329. [PubMed: 974223]

Axelrod D, Ravdin P, Koppel DE, Schlessinger J, Webb WW, Elson EL, Podleski TR. Lateral motion of fluorescently labeled acetylcholine receptors in membranes of developing muscle fibers. Proc Natl Acad Sci USA. 1976b; 73:4594-4598. [PubMed: 1070010]

Axelrod D, Wight A, Webb W, Horwitz A. Influence of membrane lipids on acetylcholine receptor and lipid probe diffusion in cultured myotube membrane. Biochemistry. 1978; 17:3604-3609. [PubMed: 567490]

Becker, W. Advanced Time-Correlated Single Photon Counting Techniques. Berlin: Springer-Verlag; 2005.

Becker W, Bergmann A, Biskup C. Multispectral fluorescence lifetime imaging by TCSPC. Microsc Res Tech. 2007; 70:403-409. [PubMed: 17393532]

Becker W, Bergmann A, Weiss G. Lifetime imaging with the Zeiss LSM-510. Proc SPIE. 2002; 4620:30-35.

Benati E, Bellini V, Borsari S, Dunsby C, Ferrari C, French P, Guanti M, Guardoli D, Koenig K, Pellacani G, Ponti G, Schianchi S, Talbot C, Seidenari S. Quantitative evaluation of healthy epidermis by means of multiphoton microscopy and fluorescence lifetime imaging microscopy. Skin Res Technol. 2011; 17:295-303. [PubMed: 21518012]

Böhmer M, Pampaloni F, Wahl M, Rahn HJ, Erdmann R, Enderlein J. Time-resolved confocal scanning device for ultrasensitive fluorescence detection. Rev Sci Instrum. 2001; 72:4145-4152.

Booth MJ, Wilson T. Low-cost, frequency-domain, fluorescence lifetime confocal microscopy. J Microsc. 2004; 214:36-42. [PubMed: 15049866]

Brown EB, Wu ES, Zipfel W, Webb WW. Measurement of molecular diffusion in solution by multiphoton fluorescence photobleaching recovery. Biophys J. 1999; 77:2837-2849. [PubMed: 10545381]

Bugiel I, König K, Wabnitz H. Investigation of cell by fluorescence laser scanning microscopy with subnanosecond time resolution. Lasers in the Life Sciences. 1989; 3:47-53.

Buranachai C, Kamiyama D, Chiba A, Williams BD, Clegg RM. Rapid frequency-domain FLIM spinning disk confocal microscope: Lifetime resolution, image improvement and wavelet analysis. Journal of Fluorescence. 2008; 18:929-942. [PubMed: 18324453]

Cardullo RA. Theoretical principles and practical considerations for fluorescence resonance energy transfer microscopy. Methods Cell Biol. 2007; 81:479-494. [PubMed: 17519181]

Cardullo, RA.; Mungovan, RM.; Wolf, DE. Imaging membrane organization and dynamics. In: Dewey, G., editor. Biophysical and Biochemical Aspects of Fluorescence Spectroscopy. Plenum Press; New York: 1991. p. 231-260.

Cardullo RA, Wolf DE. Distribution and dynamics of mouse sperm surface galactosyltransferase: implications for mammalian fertilization. Biochemistry. 1995; 34:10027-10035. [PubMed: 7632675]

Carisey A, Stroud M, Tsang R, Ballestrem C. Fluorescence recovery after photobleaching. Methods Mol Biol. 2011; 769:387-402. [PubMed: 21748690] 
Chang, CW. PhD thesis. University of Michigan; 2009. Improving Accuracy and Precision in Biological Applications of Fluorescence Lifetime Imaging Microscopy. (http:// deepblue.lib.umich.edu/bitstream/2027.42/63765/1/chingwei_1.pdf)

Chang CW, Kumar S. Vinculin tension distributions of individual stress fibers within cell-matrix adhesions. J Cell Sci. 2013; 126:3021-3030. [PubMed: 23687380]

Chang CW, Mycek M-A. Enhancing precision in time-domain fluorescence lifetime imaging. J Biomed Opt. 2010a; 15:056013. [PubMed: 21054107]

Chang CW, Mycek M-A. Precise fluorophore lifetime mapping in live-cell, multi-photon excitation microscopy. Optics Express. 2010b; 18:8688-8696. [PubMed: 20588712]

Chang, CW.; Mycek, M-A. Quantitative Molecular Imaging in Living Cells via FLIM. In: Geddes, CD., editor. Reviews in Fluorescence 2010. New York: Springer; 2012a. p. 173-198.

Chang CW, Mycek M-A. Total variation versus wavelet-based methods for image denoising in fluorescence lifetime imaging microscopy. J Biophotonics. 2012b; 5:449-457. [PubMed: 22415891]

Chang CW, Sud D, Mycek M-A. Fluorescence lifetime imaging microscopy. Methods Cell Biol. 2007; 81:495-524. [PubMed: 17519182]

Chang CW, Wu M, Merajver SD, Mycek M-A. Physiological fluorescence lifetime imaging microscopy improves Forster resonance energy transfer detection in living cells. J Biomed Opt. 2009; 14:060502. [PubMed: 20059233]

Chen LC, Lloyd WR, Chang CW, Sud D, Mycek M-A. Fluorescence lifetime imaging microscopy for quantitative biological imaging. Methods Cell Biol. 2013; 114:457-488. [PubMed: 23931519]

Chen L-C, Lloyd WR, Kuo S, Kim HM, Marcelo CL, Feinberg SE, Mycek M-A. The potential of label-free nonlinear optical molecular microscopy to non-invasively characterize the viability of engineered human tissue constructs. Biomaterials. 2014; 35:6667-6676. [PubMed: 24854093]

Clayton AH, Hanley QS, Verveer PJ. Graphical representation and multicomponent analysis of singlefrequency fluorescence lifetime imaging microscopy data. J Microsc. 2004; 213:1-5. [PubMed: 14678506]

Conway DE, Breckenridge MT, Hinde E, Gratton E, Chen CS, Schwartz MA. Fluid shear stress on endothelial cells modulates mechanical tension across VE-cadherin and PECAM-1. Curr Biol. 2013; 23:1024-1030. [PubMed: 23684974]

Cubeddu R, Comelli D, D'Andrea C, Taroni P, Valentini G. Time-resolved fluorescence imaging in biology and medicine. J PHYS D APPL PHYS. 2002; 35:R61-R76.

Davoust J, Devaux PF, Leger L. Fringe pattern photobleaching, a new method for the measurement of transport coefficients of biological molecules. EMBO J. 1982; 1:1233-1238. [PubMed: 6821332]

Dayel MJ, Hom EF, Verkman AS. Diffusion of green fluorescent protein in the aqueous-phase lumen of endoplasmic reticulum. Biophys J. 1999; 76:2843-2851. [PubMed: 10233100]

Dubinsky JM, Loftus DJ, Fischbach GD, Elson EL. Formation of acetylcholine receptor clusters in chick myotubes: migration or new insertion? J Cell Biol. 1989; 109:1733-1743. [PubMed: 2793937]

Egaña LA, Cuevase RA, Baust TB, Parra LA, Leak RK, Hocehndoner S, Peña K, Quiroz M, Hong WC, Dorostkar MM, Janz R, Sitte HH, Torres GE. J Neurosci. 29:4592-4604. [PubMed: 19357284]

Elson EL. Fluorescence correlation spectroscopy: past, present, future. Biophys J. 101:2855-2870. [PubMed: 22208184]

Elson DS, Munro I, Requejo-Isidro J, McGinty J, Dunsby C, Galletly N, Stamp GW, Neil MAA, Lever MJ, Kellett PA, Dymoke-Bradshaw A, Hares J, French PMW. Real-time time-domain fluorescence lifetime imaging including single-shot acquisition with a segmented optical image intensifier. New J Phys. 2004; 6:180-192.

Esposito A, Gerritsen HC, Wouters FS. Optimizing frequency-domain fluorescence lifetime sensing for high-throughput applications: photon economy and acquisition speed. J Opt Soc Am A Opt Image Sci Vis. 2007; 24:3261-3273. [PubMed: 17912319]

Förster T. Intermolecular energy migration and fluorescence. Ann Phys (Leitzig). 1948; 2:55-75. 
Fritz RD, Letzelter M, Reimann A, Martin K, Fusco L, Ritsma L, Ponsioen B, Fluri E, Schulte-Merker S, Van Rheenen J, Pertz O. A Versatile Toolkit to Produce Sensitive FRET Biosensors to Visualize Signaling in Time and Space. Sci Signal. 2013; 6:rs12. [PubMed: 23882122]

Gerritsen HC, Sanders R, Draaijer A, Levine YK. Fluorescence lifetime imaging of oxygen in living cells. Journal of Fluorescence. 1997; 7:11-16.

Ghiggino KP, Harris MR, Spizzirri PG. Fluorescence lifetime measurements using a novel fiber-optic laser scanning confocal microscope. Review of Scientific Instruments. 1992; 63:2999-3002.

Grashoff C, Hoffman BD, Brenner MD, Zhou R, Parsons M, Yang MT, McLean MA, Sligar SG, Chen CS, Ha T, Schwartz MA. Measuring mechanical tension across vinculin reveals regulation of focal adhesion dynamics. Nature. 2010; 466:263-266. [PubMed: 20613844]

Hao Y, Macara IG. Regulation of chromatin binding by a conformational switch in the tail of the Ran exchange factor RCC1. J Cell Biol. 2008; 182:827-836. [PubMed: 18762580]

Hinde E, Digman MA, Welch C, Hahn KM, Gratton E. Biosensor Forster resonance energy transfer detection by the phasor approach to fluorescence lifetime imaging microscopy. Microsc Res Tech. 2012; 75:271-281. [PubMed: 21858900]

Hiraoka Y, Sedat JW, Agard DA. The use of a charge-coupled device for quantitative optical microscopy of biological structures. Science. 1987; 238:36-41. [PubMed: 3116667]

Hirvonen LM, Fest F, Suhling K. Wide-field time-correlated single-photon counting (TCSPC) lifetime microscopy with microsecond time resolution. Opt Lett. 2014; 39:5602-5605. [PubMed: 25360938]

Hoppe AD, Scott BL, Welliver TP, Straight SW, Swanson JA. N-way FRET microscopy of multiple protein-protein interactions in live cells. PLoS One. 2013; 8:e64760. [PubMed: 23762252]

Hunnicutt GR, Koppell DE, Kwitny S, Cowan AE. Cyclic 3',5'-AMP causes ADAM1/ADAM2 to rapidly diffuse within the plasma membrane of guinea pig sperm. Biol Reprod. 2008; 79:9991007. [PubMed: 18667756]

Jones AM, Danielson JAH, ManojKumar SN, Lanquar V, Gorssman G, Frommer WB. Abscisic acid dynamics in rootws detected with genetically encoded FRET sensors. eLIFE. 2014; 3:e01741. [PubMed: 24737862]

Kalab P, Soderholm J. The design of Forster (fluorescence) resonance energy transfer (FRET)-based molecular sensors for Ran GTPase. Methods. 2010; 51:220-232. [PubMed: 20096786]

Kalab P, Weis K, Heald R. Visualization of a Ran-GTP gradient in interphase and mitotic Xenopus egg extracts. Science. 2002; 295:2452-2456. [PubMed: 11923538]

Kolossov VL, Spring BQ, Sokolowski A, Conour JE, Clegg RM, Kenis PJ, Gaskins HR. Engineering redox-sensitive linkers for genetically encoded FRET-based biosensors. Exp Biol Med (Maywood). 2008; 233:238-248. [PubMed: 18222979]

Komatsu N, Aoki K, Yamada M, Yukinaga H, Fujita Y, Kamioka Y, Matsuda M. Development of an optimized backbone of FRET biosensors for kinases and GTPases. Mol Biol Cell. 2011; 22:46474656. [PubMed: 21976697]

Koppel DE, Axelrod D, Schlessinger J, Elson EL, Webb WW. Dynamics of fluorescence marker concentration as a probe of mobility. Biophys J. 1976; 16:315-1329.

Kuchibhotla KV, Lattarulo CR, Hyman BT, Bacskai BJ. Synchronous hyperactivity and intercellular calcium waves in astrocytes in Alzheimer mice. Science. 2009; 323:1211-1215. [PubMed: 19251629]

Kusumi A, Tsunoyama TA, Hirosawa KM, Kasai RS, Fujiwara TK. Tracking single molecules at work in living cells. Nature Chem Biol. 2014; 10:524-532. [PubMed: 24937070]

Lakowicz, JR. Principles of fluorescence spectroscopy. 3. Springer; New York: 2006.

Lam AJ, St-Pierre F, Gong Y, Marshall JD, Cranfill PJ, Baird MA, McKeown MR, Wiedenmann J, Davidson MW, Schnitzer MJ, Tsien RY, Lin MZ. Improving FRET dynamic range with bright green and red fluorescent proteins. Nat Methods. 2012; 9:1005-1012. [PubMed: 22961245]

Lin CC, Melo FA, Ghosh R, Suen KM, Stagg LJ, Kirkpatrick J, Arold ST, Ahmed Z, Ladbury JE. Inhibition of basal FGF receptor signaling by dimeric Grb2. Cell. 2012; 149:1514-1524. [PubMed: 22726438]

Lin HJ, Herman P, Lakowicz JR. Fluorescence lifetime-resolved pH imaging of living cells. Cytometry Part A. 2003; 52A:77-89. 
Liu W, Chen E, Zhao XW, Wan ZP, Gao YR, Davey A, Huang E, Zhang L, Crocetti J, Sandoval G, Joyce MG, Miceli C, Lukszo J, Aravind L, Swat W, Brzostowski J, Pierce SK. The scaffolding protein synapse-associated protein 97 is required for enhanced signaling through isotype-switched IgG memory B cell receptors. Sci Signal. 2012; 5:ra54. [PubMed: 22855505]

Lloyd, WR.; Chen, LC.; Mycek, MA. Fluorescence Spectroscopy. In: Morgan, SP.; Rose, FR.; Matcher, S., editors. Optical Techniques in Regenerative Medicine. CRC Press - Taylor \& Francis Group; 2013a. p. 171-203.

Lloyd, WR.; Chen, LC.; Wilson, RH.; Mycek, MA. Biophotonics: Clinical Fluorescence Spectroscopy and Imaging. In: Maitland, DJ.; Moore, JEJ., editors. Biomedical Technology and Devices Handbook. 2. CRC Press - Taylor \& Francis Group; 2013b. p. 335-358.

Lloyd WR, Wilson RH, Chang CW, Gillispie GD, Mycek MA. Instrumentation to rapidly acquire fluorescence wavelength-time matrices of biological tissues. Biomed Opt Express. 2010; 1:574586. [PubMed: 21258491]

Machacek M, Hodgson L, Welch C, Elliott H, Pertz O, Nalbant P, Abell A, Johnson GL, Hahn KM, Danuser G. Coordination of Rho GTPase activities during cell protrusion. Nature. 2009; 461:99103. [PubMed: 19693013]

Maeder CI, Hink MA, Kinkhabwala A, Mayr R, Bastiaens PI, Knop M. Spatial regulation of Fus3 MAP kinase activity through a reaction-diffusion mechanism in yeast pheromone signalling. Nat Cell Biol. 2007; 9:1319-1326. [PubMed: 17952059]

Martin-Fernandez ML, Clarke DT. Single molecule fluorescence detection and tracking in mammalian cells: The state-of-the-art and future perspectives. Int J Mol Sci. 2012; 13:14742-14765. [PubMed: 23203092]

Meng F, Sachs F. Visualizing dynamic cytoplasmic forces with a compliance-matched FRET sensor. J Cell Sci. 2011; 124:261-269. [PubMed: 21172803]

Meng F, Sachs F. Orientation-based FRET sensor for real-time imaging of cellular forces. J Cell Sci. 2012; 125:743-750. [PubMed: 22389408]

Meng F, Suchyna TM, Sachs F. A fluorescence energy transfer-based mechanical stress sensor for specific proteins in situ. Febs J. 2008; 275:3072-3087. [PubMed: 18479457]

Miyata H, Noda N, Fairbairn DJ, Oldenbourg R, Cardullo RA. Assembly of the fluorescent acrosomal matrix and its fate in fertilization in the water strider, Aquarius remigis. J Cell Physiol. 2011; 226:999-1006. [PubMed: 20857404]

Miyawaki A, Llopis J, Heim R, McCaffery JM, Adams JA, Ikura M, Tsien RY. Fluorescent indicators for Ca2+ based on green fluorescent proteins and calmodulin. Nature. 1997; 388:882-887. [PubMed: 9278050]

Mueller F, Morisaki T, Mazza D, McNally JG. Minimizing the impact of photoswitching of fluorescent proteins on FRAP analysis. Biophys J. 2012; 102:656-665.

Mullineaux CW, Kirchhoff $\mathrm{H}$. Using fluorescence recovery after photobleaching to measure lipid diffusion in membranes. Methods Mol Biol. 2007; 400:267-275. [PubMed: 17951740]

Munnelly HM, Roess DA, Wade WF, Barisas BZG. Interferometric fringe fluorescence photobleaching recovery interrogates entire cell surfaces. Biophys J. 1998; 75:1131-1138. [PubMed: 9675214]

Murakoshi H, Wang H, Yasuda R. Local, persistent activation of Rho GTPases during plasticity of single dendritic spines. Nature. 2011; 472:100-104. [PubMed: 21423166]

Nakamura T, Kurokawa K, Kiyokawa E, Matsuda M. Analysis of the spatiotemporal activation of rho GTPases using Raichu probes. Methods Enzymol. 2006; 406:315-332. [PubMed: 16472667]

Nakaya M, Kitano M, Matsuda M, Nagata S. Spatiotemporal activation of Rac1 for engulfment of apoptotic cells. Proc Natl Acad Sci U S A. 2008; 105:9198-9203. [PubMed: 18591655]

Nguyen AW, Daugherty PS. Evolutionary optimization of fluorescent proteins for intracellular FRET. Nat Biotechnol. 2005; 23:355-360. [PubMed: 15696158]

Ott G, Shirkey N, Haimo LT, Cardullo RA, Thaler CD. Germ cell hub position in a Heteropteran testis correlates with the sequence and location of spermatogenesis and production of elaborate sperm bundles. Mol Reprod Devel. 2015 In Press.

Ottolia M, Phillipson KD, John S. Conformational changes of the $\mathrm{Ca}^{2+}$ regulatory site of the $\mathrm{Na}^{+}-$ $\mathrm{Ca}^{2+}$ exchanger detected by FRET. Biophys J. 2004; 87:899-906. [PubMed: 15298897] 
Ouyang M, Lu S, Li XY, Xu J, Seong J, Giepmans BN, Shyy JY, Weiss SJ, Wang Y. Visualization of polarized membrane type 1 matrix metalloproteinase activity in live cells by fluorescence resonance energy transfer imaging. J Biol Chem. 2008; 283:17740-17748. [PubMed: 18441011]

Owen DM, Auksorius E, Manning HB, Talbot CB, de Beule PA, Dunsby C, Neil MA, French PM. Excitation-resolved hyperspectral fluorescence lifetime imaging using a UV-extended supercontinuum source. Opt Lett. 2007; 32:3408-3410. [PubMed: 18059949]

Padilla-Parra S, Tramier M. FRET microscopy in the living cell: different approaches, strengths and weaknesses. Bioessays. 2012; 34:369-376. [PubMed: 22415767]

Pelet S, Previte MJ, So PT. Comparing the quantification of Forster resonance energy transfer measurement accuracies based on intensity, spectral, and lifetime imaging. J Biomed Opt. 2006; 11:34017. [PubMed: 16822067]

Provenzano PP, Eliceiri KW, Keely PJ. Multiphoton microscopy and fluorescence lifetime imaging microscopy (FLIM) to monitor metastasis and the tumor microenvironment. Clin Exp Metastasis. 2009; 26:357-370. [PubMed: 18766302]

Redford GI, Clegg RM. Polar plot representation for frequency-domain analysis of fluorescence lifetimes. Journal of Fluorescence. 2005; 15:805-815. [PubMed: 16341800]

Sakai R, Repunte-Canonigo V, Raj CD, Knopfel T. Design and characterization of a DNA-encoded, voltage-sensitive fluorescent protein. Eur J Neurosci. 2001; 13:2314-2318. [PubMed: 11454036]

Sanders R, Draaijer A, Gerritsen HC, Houpt PM, Levine YK. Quantitative pH imaging in cells using confocal fluorescence lifetime imaging microscopy. Analytical Biochemistry. 1995; 227:302-308. [PubMed: 7573951]

Schlessinger J, Koppel DE, Axelrod D, Jacobson K, Webb WW, Elson EL. Lateral transport on cell membranes: mobility of concanavalin A receptors on myoblasts. Proc Natl Acad Sci USA. 1976; 73:2409-2413. [PubMed: 1065895]

Schlessinger J, Axelrod D, Koppel DE, Webb WW, Elson EL. Lateral transport of a lipid probe and labeled proteins on a cell membrane. Science. 1977; 195:307-309. [PubMed: 556653]

Sharman KK, Periasamy A, Ashworth H, Demas JN, Snow NH. Error analysis of the rapid lifetime determination method for double-exponential decays and new windowing schemes. Analytical Chemistry. 1999; 71:947-952. [PubMed: 21662765]

Shimozono S, Hosoi H, Mizuno H, Fukano T, Tahara T, Miyawaki A. Concatenation of cyan and yellow fluorescent proteins for efficient resonance energy transfer. Biochemistry. 2006; 45:62676271. [PubMed: 16700538]

Sinnecker D, Voigt P, Hellwig N, Schaefer M. Reversible photobleaching of enhanced green fluorescent proteins. Biochemistry. 2005; 44:7085-7094. [PubMed: 15865453]

Skala MC, Riching KM, Gendron-Fitzpatrick A, Eickhoff J, Eliceiri KW, White JG, Ramanujam N. In vivo multiphoton microscopy of NADH and FAD redox states, fluorescence lifetimes, and cellular morphology in precancerous epithelia. Proc Natl Acad Sci U S A. 2007; 104:19494-19499. [PubMed: 18042710]

Sprague BL, Pego RL, Stavreva DA, McNally JG. Analysis of binding reactions by fluorescence recovery after photobleaching. Biophys J. 2004; 86:3473-3495. [PubMed: 15189848]

Spring BQ, Clegg RM. Image analysis for denoising full-field frequency-domain fluorescence lifetime images. J Microsc. 2009; 235:221-237. [PubMed: 19659915]

Stirnweiss A, Hartig R, Gieseler S, Lindquist JA, Reichardt P, Philipsen L, Simeoni L, Poltorak M, Merten C, Zuschratter W, Prokazov Y, Paster W, Stockinger H, Harder T, Gunzer M, Schraven B. $\mathrm{T}$ cell activation results in conformational changes in the Src family kinase Lck to induce its activation. Sci Signal. 2013; 6:ra13. [PubMed: 23423439]

Sud D, Mehta G, Mehta K, Linderman J, Takayama S, Mycek M-A. Optical imaging in microfluidic bioreactors enables oxygen monitoring for continuous cell culture. J Biomed Opt. 2006a; 11:050504. [PubMed: 17092147]

Sud D, Zhong W, Beer DG, Mycek MA. Time-resolved optical imaging provides a molecular snapshot of altered metabolic function in living human cancer cell models. Optics Express. 2006b; 14:4412-4426. [PubMed: 19516593]

Sud D, Mycek M-A. Calibration and validation of an optical sensor for intracellular oxygen measurements. J Biomed Opt. 2009; 14:020506. [PubMed: 19405711] 
Sun Y, Wallrabe H, Booker CF, Day RN, Periasamy A. Three-color spectral FRET microscopy localizes three interacting proteins in living cells. Biophys J. 2010; 99:1274-1283. [PubMed: 20713013]

Tsutsui H, Karasawa S, Okamura Y, Miyawaki A. Improving membrane voltage measurements using FRET with new fluorescent proteins. Nat Methods. 2008; 5:683-685. [PubMed: 18622396]

Urayama, PK.; Mycek, MA. Fluorescence lifetime imaging microscopy of endogenous biological fluorescence. In: Mycek, MA.; Pogue, BW., editors. Handbook of Biomedical Fluorescence. New York: Marcel Dekker, Inc; 2003. p. 211-236.

Verma D, Ye N, Meng F, Sachs F, Rahimzadeh J, Hua SZ. Interplay between cytoskeletal stresses and cell adaptation under chronic flow. PLoS One. 2012; 7:e44167. [PubMed: 23028495]

Vonesch, C. PhD thesis. ÉCOLE POLYTECHNIQUE FÉDÉRALE DE LAUSANNE; 2009. Fast and automated wavelet-regularized image restoration in fluorescence microscopy.

Vonesch C, Unser M. A fast multilevel algorithm for wavelet-regularized image restoration. IEEE Trans Image Process. 2009; 18:509-523. [PubMed: 19188124]

Waadt R, Hitomi K, Nishimura N, Hitomi C, Adams SR, Getzoff ED, Schroeder JI. FRET-based receptors for the direct visualization of abscisic acid concentration changes and distribution in Arabidopsis. eLife. 2014; 3:e01739. [PubMed: 24737861]

Waharte F, Brown CM, Coscoy S, Coudrier E, Amblard F. A Two-Photon FRAP analysis of the cytoskeleton dynamics in the microvilli of intestinal cells. Biophs J. 2005; 88:1467-1478.

Wang XF, Uchida T, Coleman DM, Minami S. A two-dimensional fluorescence lifetime imaging system using a gated image intensifier. Applied Spectroscopy. 1991; 45:360-366.

Waters JC. Live-cell fluorescence imaging. Methods Cell Biol. 2007; 81:115-140. [PubMed: 17519165]

Wolf DE, Lipscomb AC, Maynard VM. Causes of nondiffusing lipid in the plasma membrane of mammalian spermatozoa. Biochemistry. 1988; 27:860-865. [PubMed: 2835079]

Wolf DE, Scott BK, Millette CF. The development of regionalized lipid diffusibility in the germ cell plasma membrane during spermatogenesis in the mouse. J Cell Biol. 1986; 103:1745-1750. [PubMed: 3782284]

Wolf DE, Voglmayr JK. Diffusion and regionalization in membranes of maturing ram spermatozoa. J Cell Biol. 1984; 98:1678-1684. [PubMed: 6725394]

Wright BK, Andrews LM, Markham J, Jones MR, Stringari C, Digman MA, Gratton E. NADH distribution in live progenitor stem cells by phasor-fluorescence lifetime image microscopy. Biophys J. 2012; 103:L7-9. [PubMed: 22828352]

Xie Y, Ottolia M, John SA, Chen J-N, Philipson KD. Conformational changes of a $\mathrm{Ca}^{2+}$-binding domain of the $\mathrm{Na}^{+} / \mathrm{Ca}^{2+}$ exchanger monitored by FRET in transgenic zebrafish heart. Am J Cell Physiol. 2008; 295:C388-C393.

Zhang J, Allen MD. FRET-based biosensors for protein kinases: illuminating the kinome. Mol Biosyst. 2007; 3:759-765. [PubMed: 17940658]

Zhong W, Wu M, Chang CW, Merrick KA, Merajver SD, Mycek MA. Picosecond-resolution fluorescence lifetime imaging microscopy: a useful tool for sensing molecular interactions in vivo via FRET. Optics Express. 2007; 15:18220-18235. [PubMed: 19551120]

Zimmermann G, Papke B, Ismail S, Vartak N, Chandra A, Hoffmann M, Hahn SA, Triola G, Wittinghofer A, Bastiaens PI, Waldmann H. Small molecule inhibition of the KRAS-PDEdelta interaction impairs oncogenic KRAS signalling. Nature. 2013; 497:638-642. [PubMed: 23698361] 


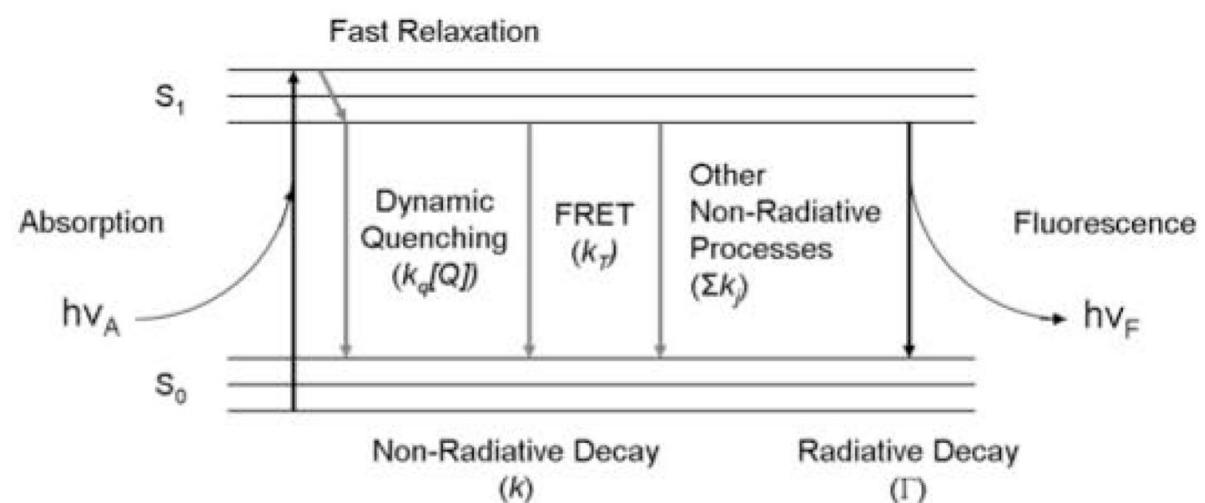

Figure 1. A simplified Jablonski diagram illustrating the fluorescence process

Photons that are absorbed by fluorochromes undergo a number of different processes including the emission of a photon $\left(\mathrm{hv}_{\mathrm{F}}\right)$ of lower energy than the photon that is absorbed $\left(h v_{A}\right.$. ). $S_{0}$ and $S_{1}$ are the ground and first-excited electronic states, respectively, and the horizontal lines represent different vibrational states of the fluorochrome. In condensed phases, following light absorption $\left(\mathrm{hv}_{\mathrm{A}}\right)$, almost all molecules rapidly relax to the lowest vibrational state of the $S_{1}$ state, from which molecules return to the ground state via one of two decay processes: non-radiative or radiative decay. The radiative decay rate $(\Gamma)$ depends on the electronic properties of an isolated fluorochrome. Molecular interactions, such as dynamic (or collisional) quenching and energy transfer, are treated in the non-radiative decay rate $(k)$. Radiative decay is responsible for fluorescence emission $\left(\mathrm{hv}_{\mathrm{F}}\right)$, providing detectable photons. $k_{q}$, bimolecular quenching constant; $[Q]$, quencher concentration; $k_{T}$, energy transfer rate constant; $k_{j}$, rate constant for non-radiative processes other than dynamic quenching and FRET (Chang et al., 2007). 


\section{Fluorescence Recovery After Photobleaching}

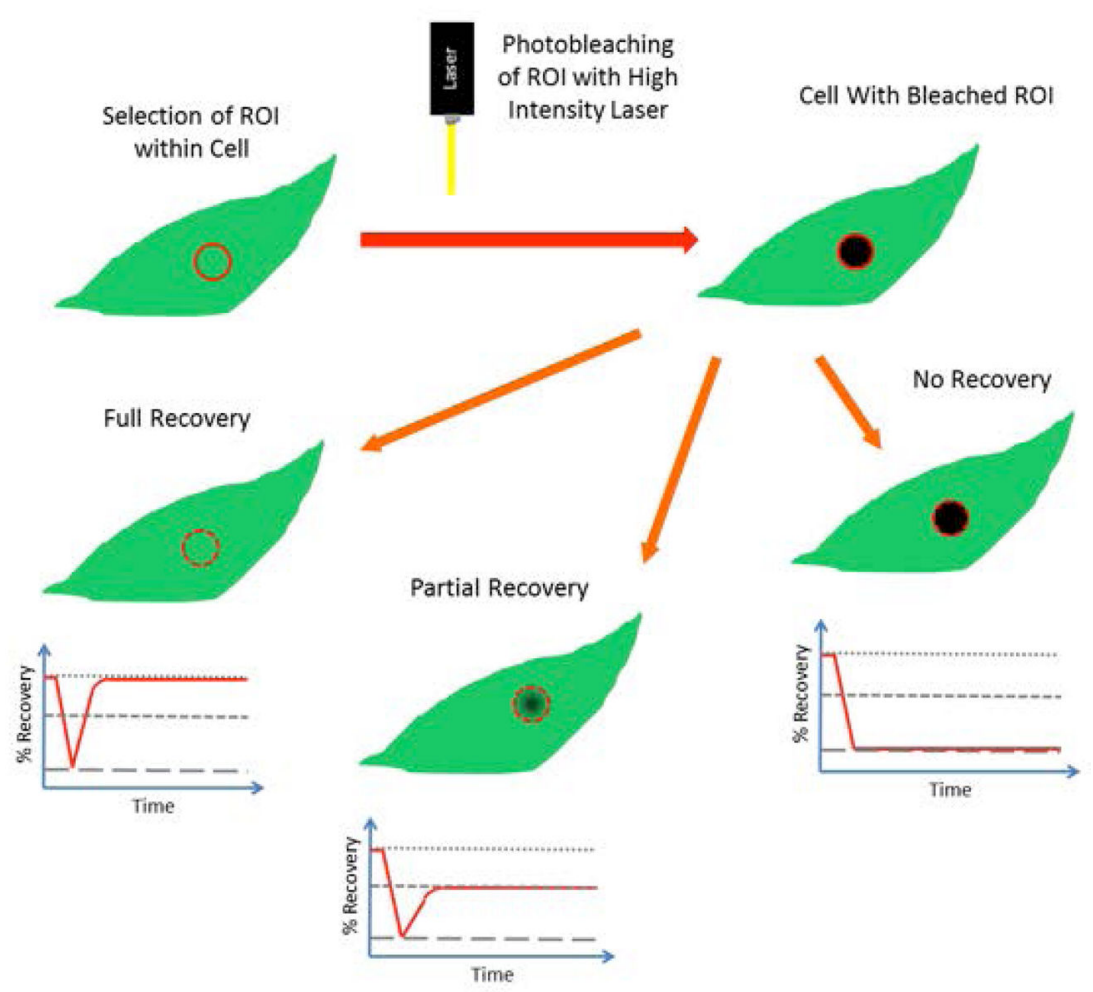

Figure 2. Principles of FRAP

FRAP can be broken down into three steps: Pre-bleach, bleach, and post-bleach. In the prebleach step, the entire sample is illuminated using a low intensity light source in order obtain a background reference signal. In the bleach step, the molecules within the region of interest are photobleached using a high-intensity laser for a brief period of time (typically $\leq 20$ $\mathrm{msec}$ ). During the post-bleach step, the sample is illuminated with a low-intensity light source, and the movement of bleached molecules out of the original bleach spot and the movement of fluorescent molecules into the original bleach spot are monitored. The postbleach step provides information about the fractional recovery (three possibilities are shown), and the diffusion coefficient of the mobile fraction (often taken from the half-time for recovery). 

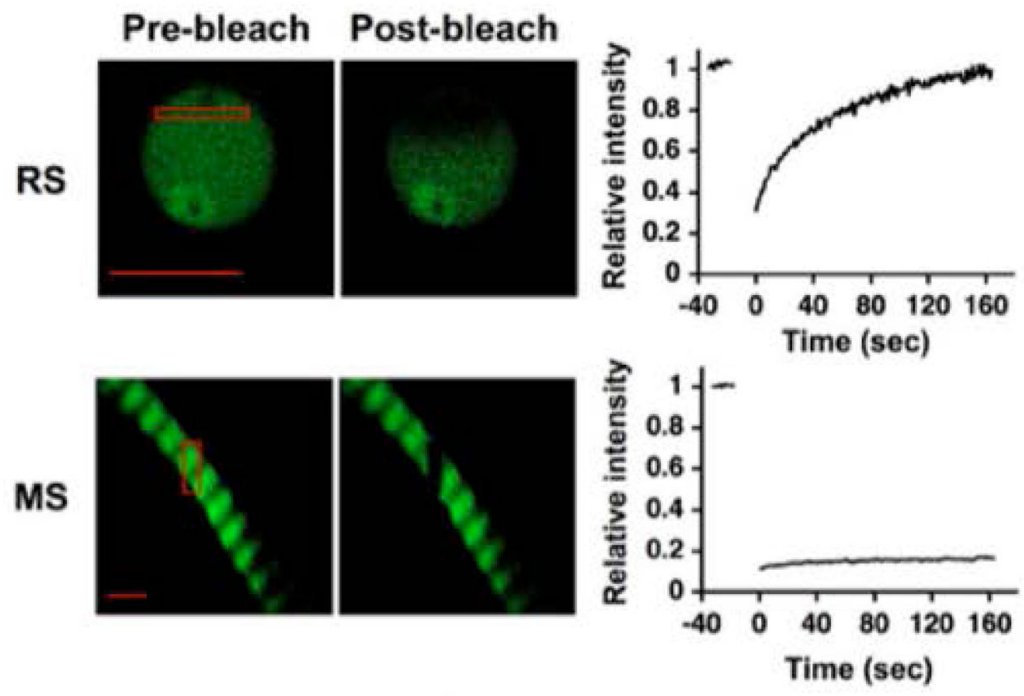

Figure 3. Use of FRAP to document the assembly of a fluorescent acrosomal matrix during spermiogenesis

The acrosomal matrix of sperm from the water strider, Aquarius remigis, is formed in postmeiotic spermatogenic cells. Pre-bleach and post-bleach images of round spermatids (RS) and mature sperm bundles (MS) are shown; the red rectangles represent the areas that were photobleached. The recovery curves document that flavin adenine dinucleotide (FAD)conjugated monomers are freely diffusing in round spermatids, but become immobilized into a rigid helical matrix in mature sperm. Reprinted with permission from Miyata et al., (2011). 
A.
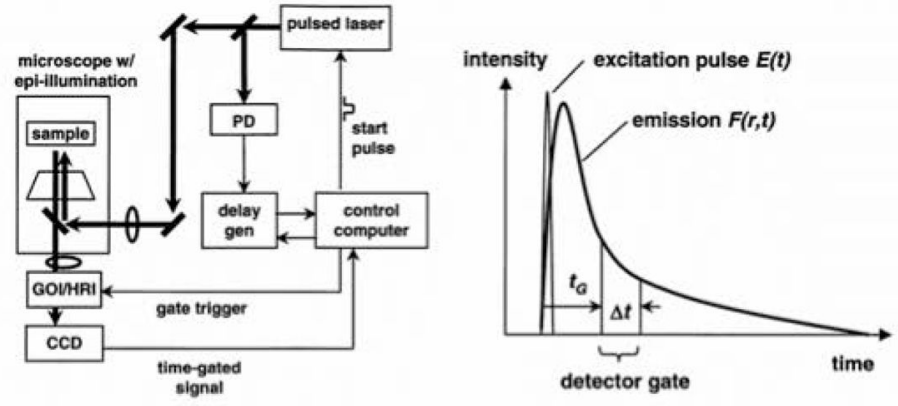

B.
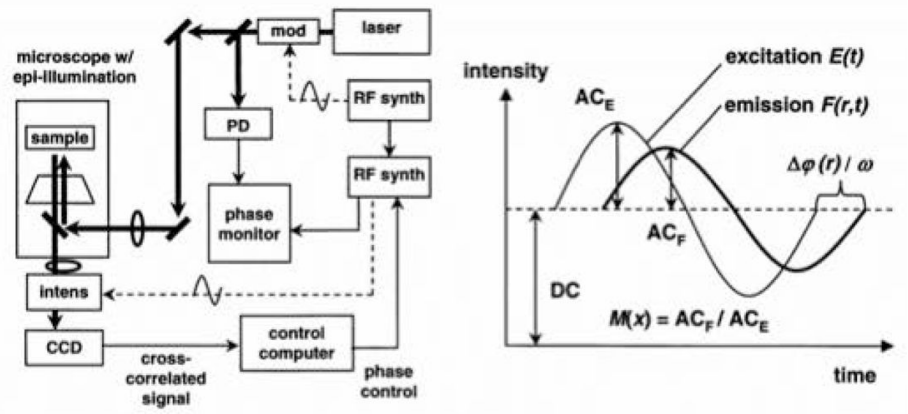

Fig. 4. FLIM instrumentation design

Time-domain (A) and frequency-domain (B) lifetime imaging instrumentation and concepts. The variable $r$ indicates parameters that are spatially varying. PD, photodiode; CCD, chargecoupled device (camera); RF synth, RF synthesizer; mod, intensity modulator; thick solid lines, light path; thin solid line, electronic path; dashed line, RF signal path. Reprinted with permission from Urayama and Mycek (2003). 

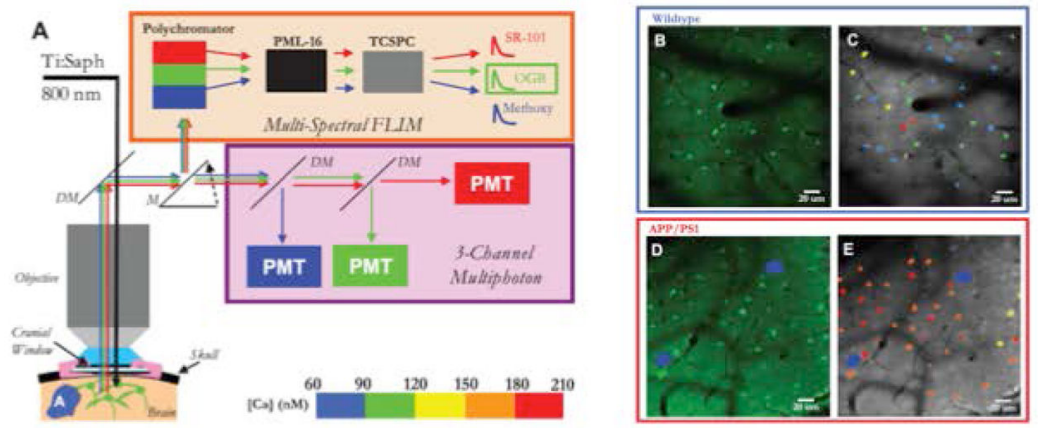

Figure 5. In vivo multi-photon FLIM detection of globally elevated levels of resting calcium in astrocytic networks

A: Multi-photon laser illumination simultaneously excited methoxy-XO4 (blue, A $\beta$ ), OGB (green, neurons and astrocytes), and SR-101 (red, astrocytes) through a cranial window. The resulting fluorescence emission was sent to either (1) a three-channel intensity-based photomultiplier tube (PMT) module or (2) a 16-channel multispectral FLIM detector. A single-photon counter (TCSPC) recorded fluorescence lifetime data. B to E: fluorescence decay curves were fit with a calcium-bound lifetime (2359 ps) and an unbound calcium lifetime (569 ps) for each pixel. The pixel data were averaged to obtain single-cell calcium levels, depicted with a calibrated color bar (in $\mathrm{C}$ and $\mathrm{E}$ ). Figure adapted and reprinted with permission from Kuchibhotla et al. (2009). 


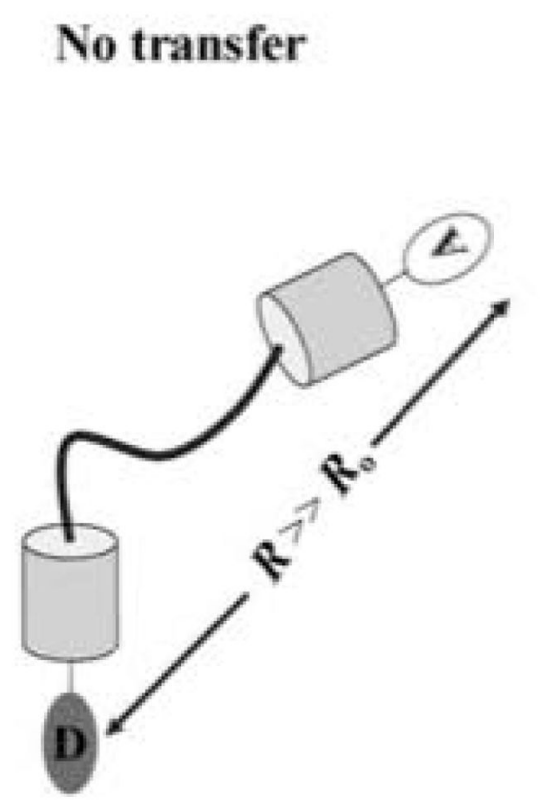

\section{Energy transfer}

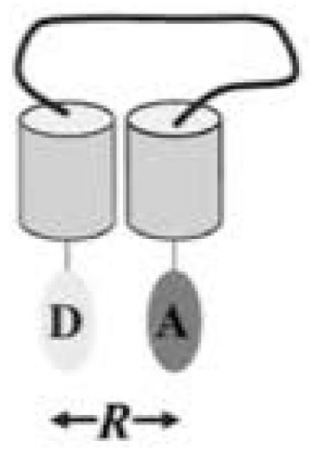

Figure 6. FRET occurs over short molecular distances

Energy transfer efficiency falls off inversely as the sixth power of the separation distance (R). The distance at which the energy transfer efficiency is $50 \%$ is defined by $\mathrm{R}_{\mathrm{O}}$, which is a characteristic of the spectroscopic properties of the donor and acceptor fluorochromes. In most cases, a separation of more than 50 Ångstroms will yield very low energy transfer efficiencies whereas separations on the order of 30 Ångstroms or less can be detected by either steady-state mechanisms (quenching of the donor fluorescence and sensitized emission of the acceptor fluorescence) or a decrease in fluorescence lifetime of the donor. 

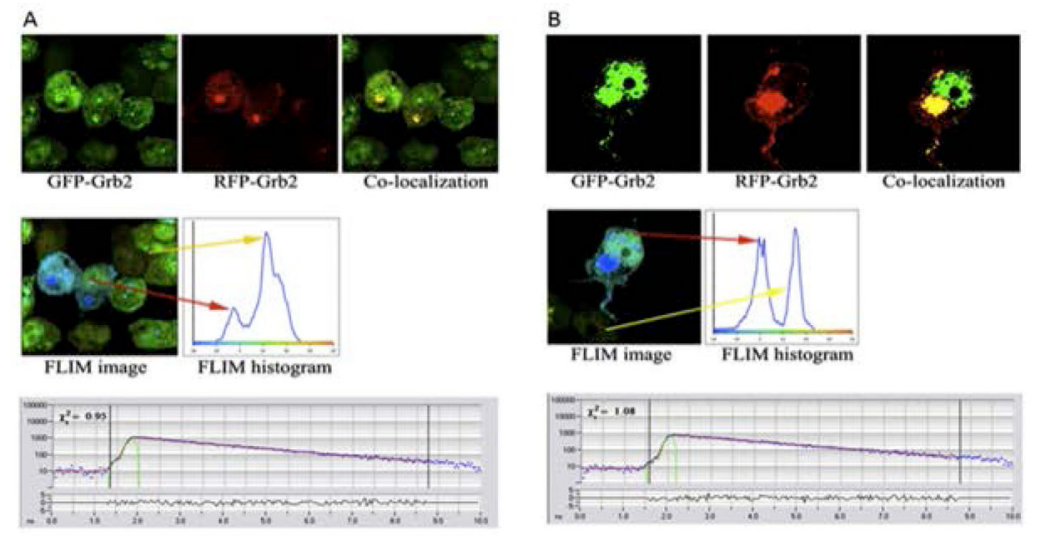

Figure 7. Detection of FRET by FLIM reveals GRB2 dimerization in the cell and on the plasma membrane

A: GFP-tagged GRB2 (GFP-Grb2, as FRET donor) and RFP-tagged GRB2 (RFP-Grb2, as FRET acceptor) were cotransfected into HEK293T cells, and the GFP lifetime was measured. Lifetime measurements revealed that the cells expressing both donor and acceptor GRB2 show a reduction in GFP lifetime due to FRET. Cells expressing only donor GRB2 show longer lifetimes in the absence of FRET, and act as an internal control for the FLIM experiments. The lifetime histogram reveals two average lifetime peaks corresponding to the shorter (GFP-GRB2/RFP-GRB2 dimer) lifetime, centered at $1.9 \mathrm{~ns}$, and the GFP-GRB2alone lifetime, centered at $2.2 \mathrm{~ns}$ (see arrows). This clearly demonstrates GRB2 can dimerize under the appropriate cellular context. Fluorescence decays were fitted with a single exponential decay model shown at the bottom. B: GRB2 dimers localize to the plasma membrane in the presence of FGFR2. In order to investigate if the dimeric GRB2 could localize to the plasma membrane in the presence of fibroblast growth factor receptor 2 (FGFR2), GFP-GRB2 and RFP-GRB2 were cotransfected together with the FGFR2 in HEK293T cells. FLIM measurement revealed that colocalized GRB2 forms dimers on the plasma membrane (see FLIM image). This confirms that GRB2 dimers are recruited to the cell membrane in the presence of FGFR2. Reprinted with permission from Lin et al. (2012). 

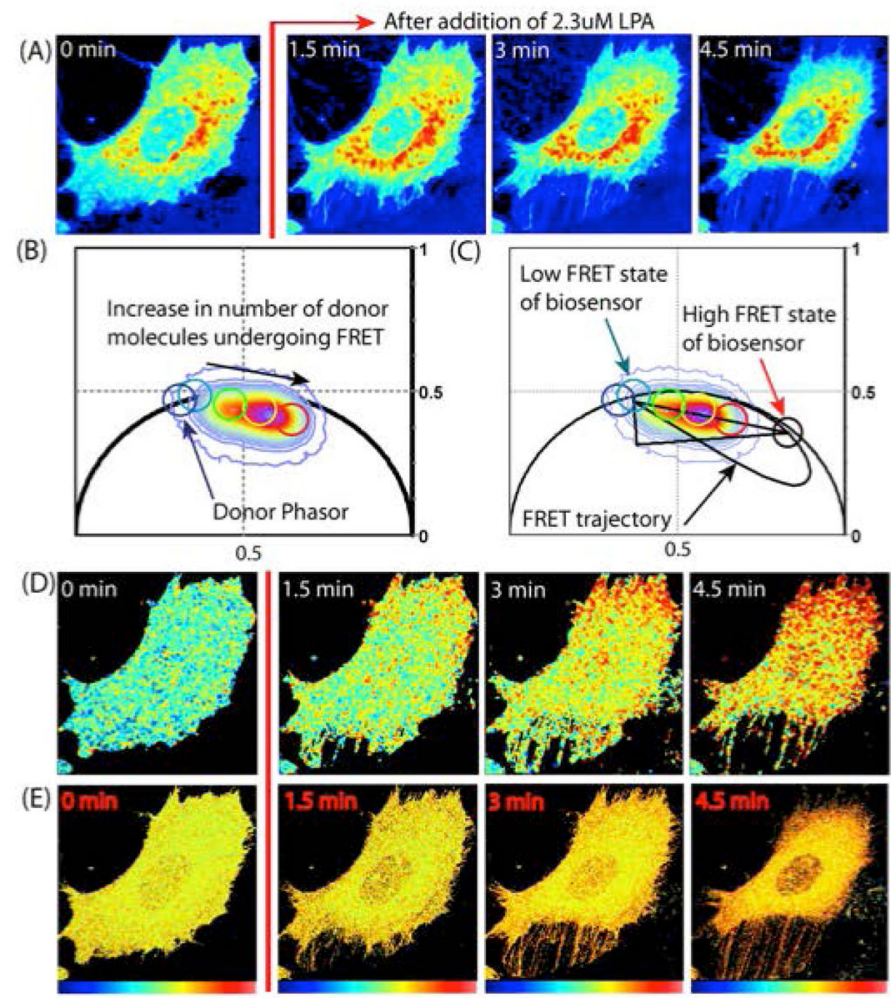

Figure 8. Phasor analysis of frequency-domain FLIM for FRET detection of a single chain RhoA biosensor, with normal sub-cellular localization

A: Intensity images of COS7 cell transfected with (RBD-Citrine)-1L-(ECFP-RhoA) before and after LPA stimulation (donor channel). B: Phasor plot of (RBD-Citrine)-1L-(ECFPRhoA) experiment. C: FRET efficiency calculator of (RBD-Citrine)-1L-(ECFP-RhoA) experiment. D: Painted FLIM image of this (RBD-Citrine)-1L-(ECFP-RhoA) experiment. E: Painted generalized polarization images of an (RBD-Citrine)-1L-(ECFP-RhoA) experiment. Reprinted with permission from Hinde et al. (2012). 

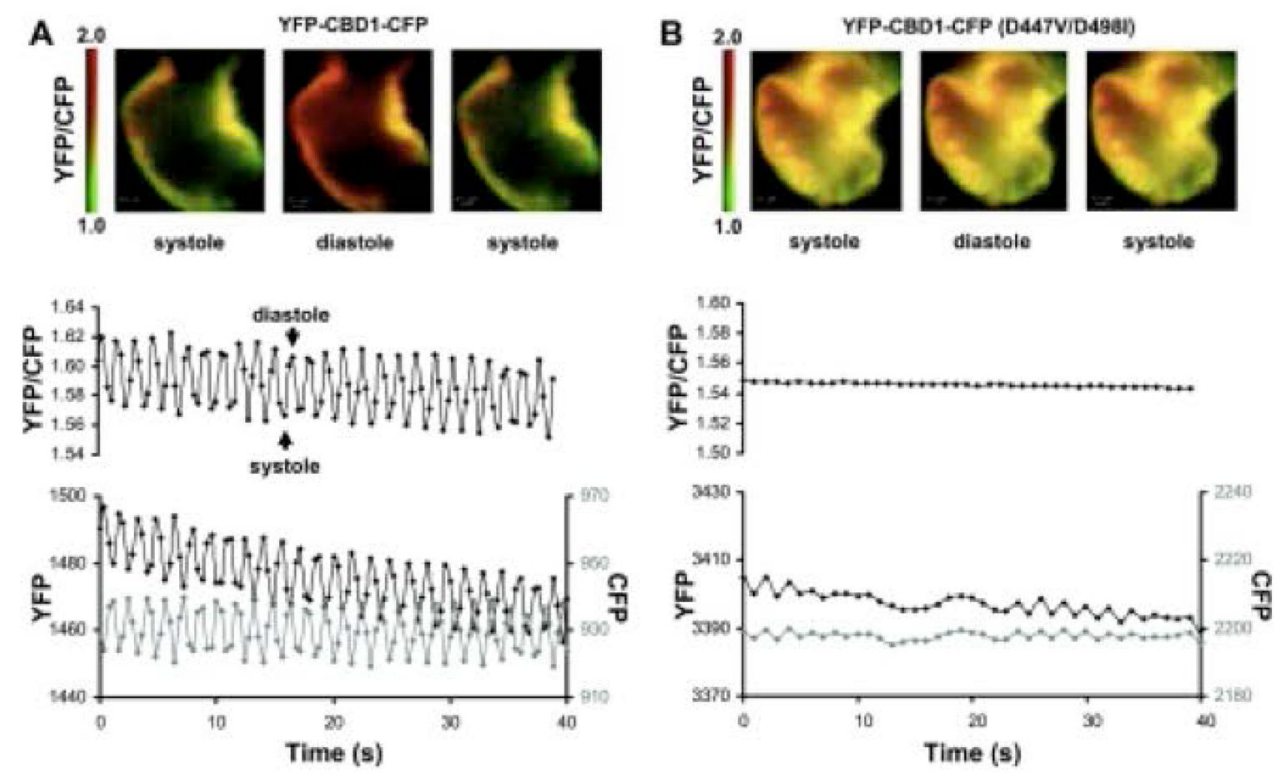

Figure 9. FRET studies in transgenic zebrafish hearts

Transgenic zebrafish expressing (A) YFP- CBD1- CFP or (B) a YFP-CBD1-CFP mutant (D447V/D498I) with decreased $\mathrm{Ca}^{2+}$ binding affinity. Representative FRET (YFP/CFP) images of transgenic zebrafish hearts (ventricle, shown in pseudocolor) and the corresponding changes in the YFP-to-CFP ratio (black, top traces), YFP (black), and CFP (gray) emissions (bottom traces) during spontaneous contractions are shown. The decrease in the ratio of YFP/CFP emissions correlates with cardiac contraction (not shown).

Pseudocolor scale bars are shown to the left of the images. Reprinted with permission from Xie et al. (2008). 
Table 1

Comparisons of time-domain FLIM and frequency-domain FLIM

\begin{tabular}{lcccc}
\hline & & Time-domain FLIM & Frequency-domain FLIM \\
\cline { 2 - 3 } & Time-gated & TCSPC & High, with wide-field microscopy \\
\cline { 2 - 4 } Data acquisition speed & High & Low & High \\
Data processing speed & High & Low, unless virtual gating is used & High \\
Multi-exponential decay resolvability & Low & High & Low \\
Large temporal range flexibility & High & High & Yes \\
Wide-field microscopy compatibility & Yes & Yes, but rare & Yes \\
Raster-scanned microscopy compatibility & Yes, but rare & Yes & Yes & No \\
Optimal (virtual) gating applicability & Yes & Yes & Yes \\
Image denoising applicability & & & \\
\hline
\end{tabular}

